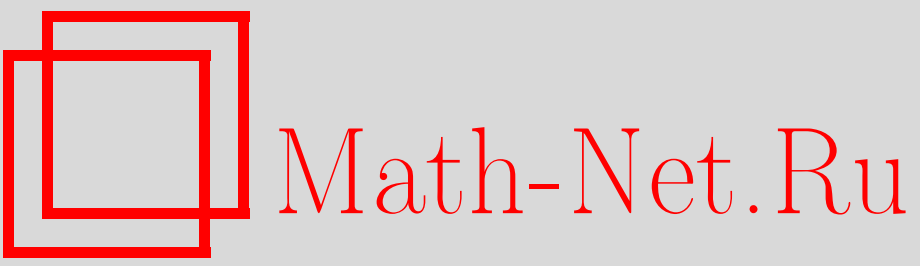

В. А. Зорич, Квазиконформные отображения и асимптотическая геометрия многообразий, УМН, 2002, том 57, выпуск 3, 3-28

DOI: https://doi.org/10.4213/rm510

Использование Общероссийского математического портала Math-Net.Ru подразумевает, что вы прочитали и согласны с пользовательским соглашением

http://www.mathnet.ru/rus/agreement

Параметры загрузки:

IP : 54.164 .48 .24

26 апреля 2023 г., 15:32:49 


\section{КВАЗИКОНФОРМНЫЕ ОТОБРАЖЕНИЯ И АСИМПТОТИЧЕСКАЯ ГЕОМЕТРИЯ МНОГООБРАЗИЙ}

\section{В. А. Зорич}

В статье-обзоре рассказано о некоторых вопросах и резултатах теории квазиконформных отображений (в основном пространственной теории и в основном в геометрическом аспекте). Прослежено развитие идей от первоисточников до их современной реализации и указан ряд новых задач, естественно выросших из найденных решений старых.

Библиографоя: 113 названий.

\section{СОДЕРЖАНИЕ}

1. Квазиконформное отображение .................................. 4

2. Квазиконформные отображения в многомерном случае ............. 7

3. Теорема о глобальном гомеоморфизме ............................ 9

Асимптотика допустимого роста коэффициента квазиконформности ...... 9

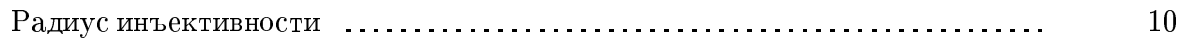

4. Переход к римановым многообразиям …....................... 10

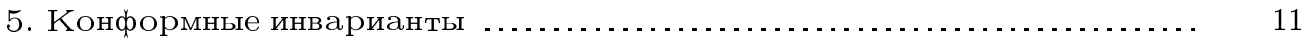

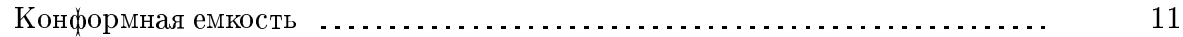

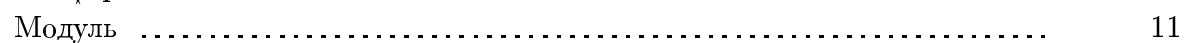

Множества нулевой емкости ….................................. 12

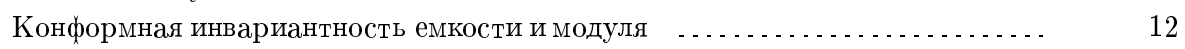

6. Конформный тип риманова многообразия ....................... 12

7. Асимптотическая геометрия и конформный тип многообразия ........ 14

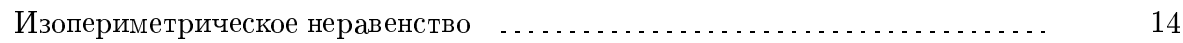

Двусторонняя оценка конформной емкости конденсатора и признаки конформного типа многообразия

Приведение к линейному виду изопериметрической функции на многообразии

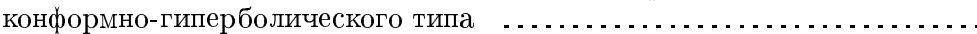

8. Конформно-инвариантная форма теоремы о глобальном гомеомор-

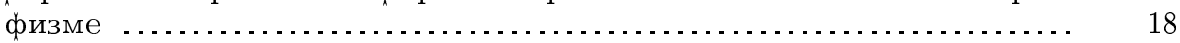

Изолированная особенность ……

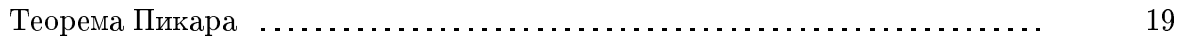

9. Некоторые открытые вопросы ................................ 20

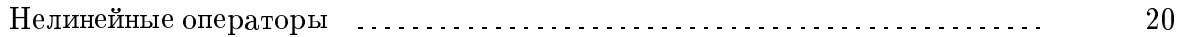

(C) В. А. Зорич 2002 
Асимптотика радиуса инъективности ........................... 20

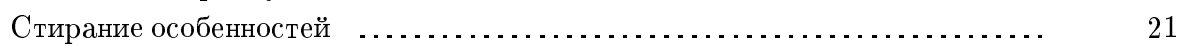

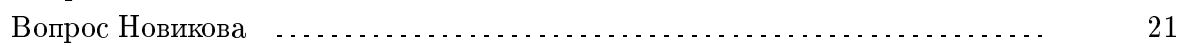

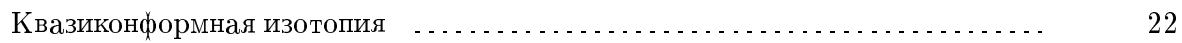

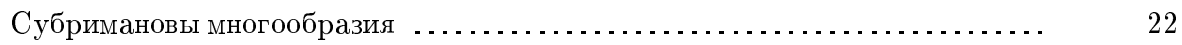

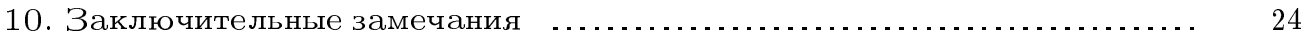

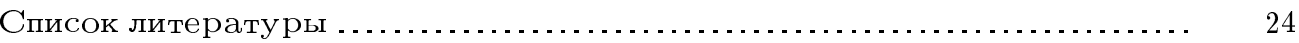

В статье рассказано о некоторых задачах и результатах близкой мне части математики. Каждый, кто упомянут в посвящении, внес в эту область свой, возможно разный по масштабам, но запоминаюшийся этапный вклад, стимулировавший дальнейшие, в том числе и мои, исследования. Изложение будет по возможности в хронологической последовательности. Это позволит проследить, как решение одних проблем порождает много новых (занятия наукой - дело продуктивное).

\section{1. Квазиконформное отображение}

Образом шара при невырожденном линейном отображении $L: \mathbb{R}^{n} \rightarrow \mathbb{R}^{n}$ является эллипсоид, который сам является шаром в точности тогда, когда $L$ - конформное (сохраняющее углы) отображение. Значит, отношение $k_{L}:=\lambda_{n} / \lambda_{1}$ наибольшей полуоси эллипсоида-образа к его наименьшей полуоси (или, скорее, $\log k_{L}$ ) может служить мерой отклонения отображения от конформности. Величина $k_{L}$ назьвается коэффиииентом квазиконформности отображсения $L$. (Заметим, и это нам будет полезно ниже, что на языке теории операторов $\left.k_{L}=\|L\| \cdot\left\|L^{-1}\right\|.\right)$

Если теперь $f$ - диффеоморфизм области $D \subset \mathbb{R}^{n}$ на область $f(D) \subset \mathbb{R}^{n}$, то, рассмотрев в каждой точке $x \in D$ касательное отображение $f^{\prime}(x)$, можно подсчитать величину $k_{f}(x):=k_{f^{\prime}(x)}$, называемую коэффициентом квазиконформности отображения $f$ в точке $x \in D$. Верхняя грань этой величины в области $D$ назьвается коэффициентом квазиконформности отображения $f$ в области $D$ и обозначается $k_{f}$. Если $k_{f}<\infty$, то говорят, что $f$-отображсение с ограниченным искажением или квазиконформное отображкение. Если $k_{f} \leqslant k$, то говорят, что $f-k$-квазиконформное отображение. Например, 1-квазиконформное отображение - конформно.

Ясно, что коэффищиент квазиконформности отображения $f$ в точке и все дальнейшее можно было определить, минуя $f^{\prime}$, используя лишш метрики исходного пространства и пространства, куда идет отображение:

$$
k_{f}(x):=\limsup _{\delta \rightarrow+0} \frac{\sup \left\{d_{Y}(f(\xi), f(x)) \mid d_{X}(\xi, x)=\delta\right\}}{\inf \left\{d_{Y}(f(\xi), f(x)) \mid d_{X}(\xi, x)=\delta\right\}}
$$

Ясно также, что данное определение применимо и к неоднолистным отображениям, например, к локальным диффеоморфизмам.

Наконец, видно, что введенные величины и понятия инвариантны относительно любых зависяших только от точки, но не от направления, изменений масштабов (что физики относят к разряду калибровочных преобразований, а геометры назьвают конформной заменой метрики).

Работа выполнена при поддержке Российского фонда фундаментальных исследований (грант № 02-01-01291). 
Квазиконформные отображения появились в конце 20-х - начале 30-х годов минувшего столетия в работах Грётша [1], [2] и Лаврентьева [3], [4] (хотя неявно присутствовали уже у Гаусса и Бельтрами в связи с введением изотермических, т.е. конформно-евклидовых, координат на двумерной поверхности). Было обнаружено [1], [4], что многие, даже сравнительно тонкие факты комплексного анализа (например, теорема Пикара) верны вовсе не только для голоморфных функций (подобно тому, как "основная теорема алгебры" о существовании корня комплексного полинома есть очень важный и очень специальный случай теоремы об индексе отображения). Это обстоятельство нашло свое далеко идущее развитие в исследованиях Альфорса 30-х годов, построившего геометрический аналог теории Неванлинны распределения значений голоморфных функций [5]. За исследования по комплексному анализу он стал в 1936 году (наряду с Дугласом, награжденньм за результаты по проблеме Плато) одним из двух первых лауреатов премии Филдса. Именно у Альфорса [5], по-видимому, и появился сам термин квазиконформное отображение.

В конще 30-х - начале 40-х годов квазиконформные отображения оказываются в центре программы Тейхмюллера [6]-[9] исследования проблемь модулей римановых поверхностей, идущей от Абеля и Римана. На одной и той же топологической поверхности, например, на компактной поверхности рода g могут быть реализованы разные конформные структуры. Сколькими параметрами (модулями) определяется это разнообразие конформных структур и как ввести эти параметры (желательно с комплексной структурой на них) - вот содержание проблемы модулей. В упрошенном виде это вопрос о том, сколько конформно различных римановых поверхностей данного рода $g$ бьвает. Ответ: при $g>1$ их разнообразие определяют $6 g-6$ вешественных или $3 g-3$ комплексных параметров. Тейхмюллер ввел расстояние (метрику Тейхмюллера) между различными римановьми поверхностями данного рода (т.е. между конформными структурами или точками пространства параметров) следующим образом. Если рассматриваемые поверхности одного рода $g$ не эквивалентны конформно, то во всяком случае они диффеоморфны. Всякий диффеоморфизм компакта является квазиконформньм отображением. Пусть $k$ - коэффициент квазиконформности экстремального квазиконформного отображения, наименее уклоняюшегося от конформности. Тогда $\log k$ примем за рассматриваемое расстояние. Так (с некоторьми опушенными здесь уточнениями) возникает пространство Тейхмюллера римановых поверхностей.

Стоит отметить, что первая задача об отыскании наименее неконформного отображения между конформно различными объектами (играющая в этих вопросах роль, подобную роли вездесущей леммы Шварца в классической теории аналитических функций) была рассмотрена уже Грётшем [2], носит его имя и состоит в следующем. На плоскости даны два прямоугольника. Среди всех отображений этих прямоугольников с соответствием вершин найти наименее неконформное. Грётш (кстати, ученик Кёбе) доказал, что во всех разумных смысллах экстремальным оказывается соответствующее линейное отображение.

Программа Тейхмюллера (погибшего во время второй мировой войны) в значительной степени была реанимирована и реализована трудами Альфорса и Берса [10]. В пространстве Тейхмюллера была введена также комплексная структура.

В последнее время пространство Тейхмюллера стало атрибутом физиков-теоретиков, занимающихся квантовой теорией поля и так назьваемой теорией струн.

Важньм и часто используемым инструментом теории квазиконформных отобра- 
жений в вешественно двумерном случае является теорема о возможности отобразить одну область плоскости на другую решениями уравнения Бельтрами $f_{\bar{z}}=\mu(z) f_{z}$, где $|\mu|(z) \leqslant q<1$. При $\mu(z) \equiv 0$ это уравнение (система) Бельтрами сводится к уравнению (системе) Коши-Римана $f_{\bar{z}}=0$, а сама теорема - к классической теореме Римана о конформном отображении областей плоскости. Заметим, что вопрос о конформном отображении двумерной поверхности, например, графика функции, на плоскую область (а значит, и вопрос о конформно-евклидовых, или изотермических, координатах на поверхности) немедленно приводится именно к уравнению Бельтрами. Действительно, бесконечно малые круги в точках (точнее, в касательных плоскостях) изогнутой поверхности при ортогональном проектировании ее в плоскость превратятся в распределенное по плоской области семейство бесконечно малых эллипсов. Мы хотим теперь трансформировать полученную в проекции область посредством вспомогательного отображения $w=f(z)$ так, чтобы касательное отображение $d w=f_{z} d z+f_{\bar{z}} d \bar{z}$ в любой точке переводило соответствуюший эллипс в круг. А это и означает, что искомое отображение плоской области удовлетворяет уравнению Бельтрами, в котором функция $\mu(z)=f_{\bar{z}} / f_{z}$ отвечает данному распределению. Добавим, что если в уравнении Бельтрами коэффициент Бельтрами $\mu_{\zeta}(z)$ голоморфно зависит от комплексного параметра $\zeta$, то и соответствуюшим образом нормированное решение уравнения также голоморфно зависит от этого параметра [11]. (Развитие и варианты доказательства этой важной теоремы существования можно проследить по работам [4], [8], [12]-[16]. Кстати, здесь особенно отчетливо видно, что М. А. Лаврентьев воспринимал квазиконформность широко, как подчиненность некоторой весьма обшей системе уравнений или соотношений, а отображения, квазиконформные в нынешнем понимании, назьвал отображсениями с ограниченнылм искажением [13].)

Коэффициент Бельтрами, как легко видеть, связан с коэффициентом квазиконформности отображения в точке простым соотношением

$$
k_{f}(z)=\frac{1+|\mu|(z)}{1-|\mu|(z)}
$$

Квазиконформные отображения оказались тесно связанными и с комплексной $\partial и$ намикой [17]. Пусть $E$ - множество на комплекснойплоскости и $f_{\zeta}$-голоморфно зависяшее от параметра $\zeta,|\zeta|<\delta<1$, семейство инъективных отображений множества $E$ в эту же плоскость (голоморфная эволюция), причем $f_{0}$ - тождественное отображение. (Голоморфная зависимость от параметра $\zeta$ подразумевает, что при любом $z \in E$ комплексная величина $f_{\zeta}(z)$ есть голоморфная функция параметра $\zeta$.) Оказьвается, всякая такая эволюция множества $E \subset C$ продолжается до голоморфного движения всей плоскости, причем можно считать, что областью изменения параметра является единичный круг. В каждый момент $\zeta$ отображение $f_{\zeta}$ удовлетворяет некоторому уравнению Бельтрами, в котором коэффициент Бельтрами голоморфно зависит от параметра $\zeta$. При этом $\left|\mu_{\zeta}\right|(z)<1$ и по условию $\mu_{0}(z) \equiv 0$. Тогда по лемме Шварца $\left|\mu_{\zeta}\right|(z)<|\zeta|$ при любом $z \in C$ и любом $|\zeta|<1$. Таким образом, голоморфная эволюиия происходит квазиконформно, причем коэффициент квазиконформности отображения $f_{\zeta}$ оценивается сверху величиной $(1+|\zeta|) /(1-|\zeta|)$. Это позволяет теперь использовать свойства квазиконформных отображений при изучении голоморфной динамики. (См. [17] и цитируемую там литературу.) 


\section{2. Квазиконформные отображения в многомерном случае}

Все, о чем шла речь вьше, за исключением самого определения квазиконформного отображения, относилось к вешественно двумерному или комплексно одномерному случаю. Подобно тому как теория функций многих комплексных переменных наполнена специфически многомерными явлениями (они-то и представляют основной интерес), теория квазиконформных отображений в пространстве сталкивается с эффектами, которые не наблюдаются в плоском случае.

Первые специфически многомерные эффекты теории квазиконформных отображений (если не считать классической теоремы Лиувилля о конформных отображениях), по-видимому, были отмечены в статье [18] М.А. Лаврентьевым. Это исследование не получило непосредственного развития, и сформулированные в нем утверждения еще долгое время оставались недоказанными (хотя отдельные работы общего теоретико-функционального плана, относившиеся и к многомерной ситуации, появлялись $[19],[20])$.

Систематическое изучение пространственных квазиконформных отображений началось в конце 50-х - начале 60-х годов в основном в России (Лаврентьев [21]-[23], Шабат [24], [25], Решетняк [26], [27], Белинский [28]), Финляндии (Вяйсала [29], [30]) и США (Геринг [31], [32]).

(Отметим еше отдельные работы [33]-[35], стимулировавшие ряд последуюших исследований.)

Из личных впечатлений тех времен помню, что в 1960 году, оканчивая механико-математический факультет МГУ, я писал дипломную работу по теме, полученной от моего научного руководителя, Б. В. Шабата, который был прямым учеником М. А. Лаврентьева. Одним из основных результатов работы было доказательство того, что любой квазиконформный автоморфизм открытого шара продолжается на границу.

(В двумерном случае это было установлено Мори [36] и затем доведено до полного описания возникающего автоморфизма граничной окружности Бёрлингом и Альфорсом [37] - знаменитое $M$-условие.) [38].

Первые многомерные результаты на этот счет появились в работах [25], [30], [32],

Геринг [32] отметил, что продолженное отображение квазиконформно на граничной сфере.

Это обстоятельство позднее было с успехом использовано Мостовым в его красивой работе [39] о жесткости пространственных гиперболических форм (см. также [40]). Мостов, в частности, показал, что если два компактных римановых многообразия одинаковой постоянной отрицательной кривизны диффеоморфньи, а их размерность больше двух, то они изометричны.

Вспоминая теорему униформизации Клейна-Пуанкаре-Кёбе и модель Пуанкаре планиметрии Лобачевского в круге, нетрудно понять, что это находится в полном контрасте с сушествованием модулей конформных структур на двумерных поверхностях.

Теорема Мостова имела большой резонанс и развивалась во многих направлениях (в работах Маргулиса, Салливана, Тукиа, самого Мостова и других).

Однако первое наблюдение о конформной жесткости пространства было сделано еще в середине XIX-го века, когда почти одновременно Риман [41; с. 49-87] установил (1851 г.) свою теорему о богатстве конформных отображений областей размерности $n=2$, а Лиувилль [42] установил (1850г.) конформную жесткость областей пространства $\mathbb{R}^{n}, n \geqslant 3$, доказав следуюшее: 
Каждое конформное отображение (класса гладкости $C^{(4)}$ ) области пространства $\mathbb{R}^{n}$ при $n>2$ является композицией инверсий.

Таким образом, в $\mathbb{R}^{n}$ при $n \geqslant 3$ конформные отображения сводятся к группе Мёбиуca, т.е. к тому, что в плоском случае называют дробно-линейными преобразованиями.

Природа явления связана с переопределенностью системы уравнений, отвечающей условию конформности отображения, когда $n>2$. Эта система есть система Коши-Римана, когда $n=2$.

Проявления конформной жесткости пространства наблюдаются и в свойствах пространственных квазиконформных отображений, о чем еще будет сказано ниже.

Большое количество современных работ было посвящено как доказательству теоремы Лиувилля при минимальных условиях регулярности отображения, так и устойчивости в этой теореме относительно разных норм (см. [21], [26]-[28], [43]-[51] и цитированную там литературу).

Вернемся теперь еще к граничным свойствам квазиконформных отображений. Продолжая ими заниматься в аспирантские годы (1960-1963), я, в вьполненной под руководством Шабата кандидатской диссертации, в частности, описал соответствие границ, возникающее при квазиконформных отображениях шара в евклидово пространство той же размерности. Тем самым был построен пространственный аналог теории Каратеодори соответствия границ.

Суть теории Каратеодори, как известно, состоит в следующем. По теореме Римана любую односвязную область, отличную от всей плоскости, можно конформно отобразить на круг. Граница области может быть сложной, и тогда точкам окружности (границы круга) на границе области не обязаны отвечать одноточечные множества. Что же соответствует точке? Конечно, имея само конформное отображение, можно было бы перенести метрику из круга в область и провести пополнение области по этой метрике. Каратеодори придумал, как все сделать, не зная отображения, а глядя только на данную область. Он определил граничные элементы (простые концы) любой односвязной области плоскости, которые в случае жордановой границы, конечно, сводятся к геометрическим точкам. По-видимому, это была первая нетривиальная топологическая компактификация односвязных областей плоскости, делающая из любой такой области объект, гомеоморфньй замкнутому кругу. (Каратеодори коснулся многих областей математики, и не только математики. Ниже мы вскользь скажем о метрике Карно-Каратеодори, порожденной работой Каратеодори по термодинамике. Кстати, по свидетельству Лаврентьева [52; с. 251] - большого знатока конформных отображений, Каратеодори принадлежит и первое исчерпьвающее доказательство теоремы Римана, полученное им в 1913 году.)

Упомянутое вьше распространение теории Каратеодори на случай квазиконформных отображений шара [38], [53] в качестве попутного продукта имело некоторые следствия, относяшиеся, с одной стороны, к исходной классической теории и, с другой стороны, к гипотезе Лаврентьева (из работы [18]) об особенностях граничного поведения квазиконформных отображений шара в пространстве. А именно, был указан максимальный класс отображений (разумеется, включаюший конформные и квазиконформные отображения), для которого соответствие границ осушествляется по простым концам Каратеодори [53]-[55], т.е. были очерчены максимальные рамки действия классической теории. С другой стороны, и это относится уже к многомерному случаю, появилась возможность предъявлять различные области, гомеоморфные шару и не допускаюшие квазиконформное отображение на шар (см. в этой связи также ра- 
боты [25], [30], [56]). Например, на плоскости круг с выброшенным радиусом можно конформно отобразить на круг, а шар с выброшенным радиусом нельзя отобразить на шар даже квазиконформно (что укладьвается в гипотезу Лаврентьева). Однако если из шара удалить не радиус, а плошадку, примыкающую к граничной сфере, то получится область, квазиконформно эквивалентная шару (что противоречит гипотезе Лаврентьева и одновременно указывает на то, как ее формулировку следует подправить).

Однако главным из двух утверждений Лаврентьева, высказанных им в работе [18], было другое, и оно оказалось абсолютно правильным (так сложилось, что его доказательство стало предметом моей докторской диссертации, зашищенной на мехмате МГУ в 1969 году).

\section{3. Теорема о глобальном гомеоморфизме}

Теорема о глобальном гомеоморфизме для квазиконформных отображений описывает следуюшее специфически многомерное явление (сформулировано в [18] для $n=3$; доказано позднее в [57]):

Локально обратимое квазиконформное отображсение $f: \mathbb{R}^{n} \rightarrow \mathbb{R}^{n}$ nри $n>2$ обратимо глобально.

(Отметим, что вопрос о глобальной обратимости локально обратимых полиномиальных отображений $\mathbb{C}^{n}$, так называемая проблема якобиана, остается открытым. Голоморфность и квазиконформность в высших размерностях - почти ортогональные свойства.)

Отображение $z \mapsto \exp (1 / z)$ показывает, что условие $n \geqslant 3$ здесь, конечно, существенно.

Существенно также и то, что $f$ локально гомеоморфно. Это уже требует некоторой работы и демонстрируется примером отображения $f: \mathbb{R}^{3} \rightarrow \mathbb{R}^{3} \backslash\{0\}$ с ограниченньм искажением, построенного в работе [57]. (Я с благодарностью вспоминаю по этому поводу один полезньй совет П.П. Белинского.) Это двоякопериодическое отображение напоминает $\exp (z)$, но, в отличие от $\exp (z)$, оно имеет ветвления вдоль двоякопериодической системы параллельных прямых. Построенное отображение, как и $\exp (z)$, имеет пару вьпускаемых значений $(0$ и $\infty)$. В этой связи в работе [57] был поставлен вопрос о справедливости теоремы Пикара для целых (определенных во всем пространстве $\mathbb{R}^{n}$ ) отображений с ограниченным искажением. Неожиданньй ответ был получен Риккманом [58], которьй показал, что число вьпускаемых значений всегда конечно и ограничено величиной, зависящей только от коэффициента квазиконформности $k_{f}$ отображения $f$; но, в отличие от двумерного случая, это число может неограниченно расти вместе с ростом коэффициента квазиконформности отображения [59], [60].

Теорема о глобальном гомеоморфизме имеет ряд нетривиальных обобщений уже в рамках $\mathbb{R}^{n}$ (см. обзор [61]). Упомянем два.

Асимптотика допустимого роста коэффициента квазиконформности.

Пусть $f: \mathbb{R}^{n} \rightarrow \mathbb{R}^{n}(n \geqslant 3)$ - произвольное локально гомеоморфное отображение, и пусть $k(r)$ - его коэффициент квазиконформности в шаре $B^{n}(r)=\left\{x \in \mathbb{R}^{n}|| x \mid \leqslant r\right\}$. Если отображение $f$ квазиконформно, то функция $k(r)$ ограничена, но в общем случае она может расти. Оказывается (см. [62]), если $\int^{\infty} \frac{d r}{r k(r)}=\infty$, то отобажение $f$ глобально обратимо, причем эта асимптотика допустимого роста $k(r)$ точна в том 
смысле, что для любой функции $k(r)$ (неотрицательной и неубываюшей), для которой указанньй интеграл сходится, можно построить такое отображение всего пространства $\mathbb{R}^{n}$ на единичный шар, у которого коэффициент квазиконформности не больше, чем $k(r)$.

Кстати, вторая часть сформулированной теоремы (пример) действует во всех размерностях $n \geqslant 2$, как и то, что инъективное квазиконформноеотображение $\mathbb{R}^{n}(n \geqslant 2)$ в себя всегда сюръективно. Если принять во внимание конформно-евклидову модель Пуанкаре гиперболического пространства Лобачевского в шаре (круге), то можно понять, что теорема дает количественное представление о степени конформного различия пространств Евклида и Лобачевского в целом.

Вьше мы упомянули остаюшуюся пока открытой проблему якобиана. Можно было бы попробовать применить к ней эту теорему. Однако коэффициент квазиконформности полиномиального отображения растет, как правило, полиномиально, а не логарифмически, поэтому для доказательства глобальной обратимости полиномиального отображения необходимо привлекать дополнительные соображения, связанные с его спецификой.

Радиус инъективности. Мартио, Риккман и Вяйсала [63] нашли следуюшее красивое развитие теоремы о глобальном гомеоморфизме, обобшающее заодно на случай квазиконформных отображений близкий результат Джона [64], относяшийся к квазиизометриям. Пусть $Q_{k}-$ класс локально гомеоморфных отображений $f: B^{n} \rightarrow$ $\mathbb{R}^{n}$ единичного шара в $\mathbb{R}^{n}$ с коэффициентом квазиконформности $k_{f}<k$. Пусть $r_{f}-$ радиус наибольшего концентрического с $B^{n}$ шара, в котором $f$ еше инъективно. Утверждение: если $n \geqslant 3$, то $r(n, k):=\inf _{f \in Q_{k}} r_{f}>0$.

Итак, каждое такое отображение инъективно в шаре радиуса $r(n, k)$, называемого радиусом инвективности. Радиус инъективности зависит только от коэффициента квазиконформности отображения и от размерности пространства.

Радиус инъективности, очевидно, меняется пропорционально радиусу исходного шара, поэтому, заменяя здесь шар $B^{n}$ всем пространством $\mathbb{R}^{n}$, мы вновь получаем теорему о глобальном гомеоморфизме (формально получаем только инъективность, но это здесь главное, дальнейшее просто).

\section{4. Переход к римановым многообразиям}

С.П. Новиков (который, наряду с А.Г. Витушкиным и А. И. Маркушевичем, был официальным оппонентом в упомянутой вьше защите моей докторской диссертации) поставил следуюший общий вопрос. Рассмотрим квазиконформное погружение (иммерсию) $f: M^{n} \rightarrow N^{n}$ одного риманова многообразия в другое риманово многообразие той же размерности $n \geqslant 3$. Для каких $M^{n}$ и $N^{n}$ справедлива теорема о глобальном гомеоморфизме? Полного ответа на этот вопрос я не знаю и сейчас, хотя кое-что свежее и содержательное будет приведено ниже.

Громов [65], [66] распространил теорему о глобальном гомеоморфизме на квазиконформные погружения римановых многообразий и частично ответил на этот вопрос:

Eсли $f: M^{n} \rightarrow N^{n}$ - локально гомеоморфное квазиконформное отображение полного риманова многообразия $M^{n}$ конечного оббема в односвязное риманово многообразие $N^{n}$, то при $n \geqslant 3$ отображсение инбективно и хаусдорфова размерность множества $N^{n} \backslash f\left(M^{n}\right)$ равна нулю. 
Класс квазиконформных отображений инвариантен относительно конформных замен римановой метрики, поэтому сопоставление исходной теоремы о глобальном гомеоморфизме с этим ее обобщением на римановы многообразия приводит к следуюшему чисто геометрическому вопросу.

Рассмотрим некомпактное риманово многообразие $\left(M^{n}, g\right)$ и класс метрик $\widetilde{g}=\lambda^{2} g$ на нем, конформно эквивалентных его исходной метрике. Когда этот класс содержит полную метрику конечного объема?

Ответ на сформулированный вопрос (и на ряд других, как выясняется, близких вопросов) ведет к разделению некомпактных многообразий на два класса: конформно-параболические и конформно-гиперболические.

После такого замечания ответ угадьвается не более чем с одной попытки. Правильньй ответ: это можно сделать тогда и только тогда, когда $\left(M^{n}, g\right)$ - многообразие конформно-параболического типа. (Например, как $\mathbb{R}^{n}$ в исходной теореме о глобальном гомеоморфизме.)

Это (теперь почти очевидное) наблюдение было сформулировано в докладе [67] и работе $[61 ;$ с. 140], где пояснено, что его можно рассматривать как геометрическую интерпретацию одного хорошо известного свойства модулей семейств кривых (или вообще семейств мер: [33; с. 179]).

Чтобы определить и обсудить понятие конформного типа риманова многообразия, напомним определения двух взаимосвязанных конформных инвариантов.

\section{5. Конформные инварианты}

Конформная емкость. Конформной емкостью конденсатора $R\left(C_{0}, C_{1}\right)$ называется величина

$$
\operatorname{cap} R\left(C_{0}, C_{1}\right):=\inf \int_{M^{n}}|\nabla U|^{n} d v,
$$

где нижняя грань берется по всем неотрицательным непрерывным функциям (потенциалам) $U: M^{n} \rightarrow \mathbb{R}_{+}$с обобщенными производньми таким, что $\left.U\right|_{C_{0}}=0,\left.U\right|_{C_{1}}=1$.

Например, конформная емкость конденсатора $R_{r_{0}}^{r_{1}} \subset \mathbb{R}^{n}$, ограниченного сферами $C_{0}=\left\{x \in \mathbb{R}^{n}|| x \mid=r_{0}\right\}, C_{1}=\left\{x \in \mathbb{R}^{n}|| x \mid=r_{1}\right\}$, где $0<r_{0}<r_{1}$, равна

$$
\operatorname{cap} R_{r_{0}}^{r_{1}}=\omega_{n-1} \ln ^{1-n}\left(r_{1} / r_{0}\right),
$$

где $\omega_{n-1}-$ это $(n-1)$-мера (плошадь) единичной сферы в $\mathbb{R}^{n}$.

(При $n=2$ это классическая емкость, связанная с интегралом Дирихле, оператором Лапласа и ньютоновским потенциалом; конформная емкость при $n>2$, по-видимому, появилась в работе [34].)

Модуль. Конформная емкость конденсатора может быть описана несколько иначе, в терминах модуля (или экстремальной длины [68], [69], [33], [24], [70]) семейства кривых (или мер). Порой такая форма предпочтительна.

Пусть $\Gamma=\{\gamma\}$ - семейство кривых на $n$-мерном римановом многообразии $\left(M^{n}, g\right)$. Измеримая по Борелю неотрицательная функция $\rho: M^{n} \rightarrow \mathbb{R}_{+}$называется допустимой для Г, если $\int_{\gamma} \rho d s \geqslant 1$ для каждой кривой $\gamma \in \Gamma$. Конформным модулем семейства кривых Г назьвается величина

$$
\bmod \Gamma:=\inf \int_{M^{n}} \rho^{n} d v,
$$


где нижняя грань берется по всем допустимым для Г функциям.

Взаимосвязь емкости и модуля конденсатора. Если $\Gamma$ - семейство всех кривых в $R\left(C_{0}, C_{1}\right)$, соединяюших граничные компоненты $C_{0}, C_{1}$ конденсатора $R\left(C_{0}, C_{1}\right)$, то $\bmod \Gamma=\operatorname{cap} R\left(C_{0}, C_{1}\right)$. Доказательство см., например, в [71], [72]. Процесс осреднения (свертка с регулярнњм ядром) позволяет при вычислении емкости конденсатора и модуля соответствующего семейства кривых ограничиться функциями порядка гладкости самого многообразия.

Множества нулевой емкости. В определении конденсатора и его емкости в качестве $C_{0}$ и $C_{1}$ можно брать и достаточно произвольные множества, не обязательно континуумы. Если множество $E:=C_{1}$ таково, что сар $R\left(C_{0}, E\right)=0$ для некоторого ( а тогда и для любого) невырожденного и не пересекающего $E$ континуума $C_{0}$, то говорят, что множество $E$ имеет нулевую конформную емкость или, полнее, что $E$ есть мно сество конформной емкости нуль.

Это равносильно тому, что модуль семейства всех кривых, пересекающих $E$, равен нулю. Метрическая (хаусдорфова) размерность такого множества $E$ тоже равнанулю (см., например, [33]).

(Кстати, если из любого семейства кривых выбросить кривые бесконечной длины, то конформньй модуль семейства не изменится. Поэтому конформньй модуль сродни электрической проводимости системы проводов и порой его правильно называют конформной проводимостью. Это сразу порождает безошибочную интуицию в интерпретации свойств этой величины (например, ясно, что происходит, если кривые семейства удлиняются, укорачиваются или к ним добавляется еще какое-то семейство кривых). Неспрямляемых кривых среди всех кривых, конечно, больше, чем спрямляемых, но для проводимости они бесполезны и она их игнорирует так же, как вычислительная процедура игнорирует иррациональные числа - красу и гордость нашей “пополненной" вешественной прямой.)

Конформная инвариантность емкости и модуля. Локальное в $\lambda$ раз изменение элемента длины $d \widetilde{s}=\lambda(x) d s$ на римановом многообразии $\left(M^{n}, g\right)$, т.е. переход к конформно эквивалентной метрике $\widetilde{g}=\lambda^{2} g$ (что на физическом языке означает калибровочное преобразование, связанное с произволом в выборе местных масштабов), приводит к изменению в $\lambda^{n}$ раз численной величины элемента объема и в $\lambda^{-1}$ раз градиента функции, поэтому форма $|\nabla U|^{n} d v$, а вместе с ней и конформная емкость инвариантны относительно конформного изменения метрики.

Аналогично, если в соответствии с изменением в $\lambda$ раз элемента длины на $\left(M^{n}, g\right)$ заменить $\rho$ новой допустимой функцией $\lambda^{-1} \rho$, то можно увидеть, что модуль семейства кривых - тоже конформный инвариант.

Нетрудно также проверить, что конформная емкость и конформньй модуль квазиинвариантны (меняются не более чем в $k^{n-1}$ раз) при $k$-квазиконформном отображении.

Это, в частности, влечет инвариантность по отношению к любым квазиконформным отображениям свойства объекта иметь емкость нуль или модуль нуль, что будет неоднократно использовано ниже.

\section{6. Конформный тип риманова многообразия}

Приведенная выше формула для конформной емкости сферического конденсатора

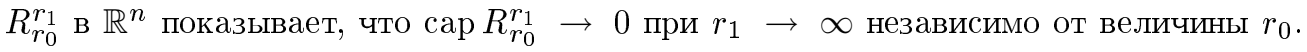


По этой причине говорят, что емкость $\mathbb{R}^{n}$ в бесконечности, или конформная емкость бесконечности пространства $\mathbb{R}^{n}$ (т.е. емкость любого конденсатора $R\left(C_{0}, C_{1}\right)$, когда $C_{1}$-компонента уведена на абсолют), равна нулю. После конформной стереографической проекции пространство $\mathbb{R}^{n}$ можно интерпретировать как проколотую сферу $\mathbb{S}^{n} \backslash p$, а точку $p \in \mathbb{S}^{n}$ как реализацию бесконечности, абсолюта или, как еще говорят, идеальной границы пространства.

Метрика Пуанкаре в модели Пуанкаре гиперболического пространства Лобачевского $H^{n}$ в единичном шаре $B^{n} \subset \mathbb{R}^{n}$ конформно-евклидова. Значит, конформные емкости гиперболических объектов можно вычислять непосредственно в евклидовой метрике. Бесконечность пространства $H^{n}$ совпадает в модели Пуанкаре с граничной сферой $\partial B^{n}$ и, в соответствии с формулой для конформной емкости сферического конденсатора $R_{r_{0}}^{r_{1}}$, имеем сар $R_{r_{0}}^{1}>0$ при любом $\left.r_{0} \in\right] 0,1[$. Таким образом, в случае пространства $H^{n}$ емкость его бесконечности (абсолюта, идеальной границы) положительна.

В классической теории римановых поверхностей, когда любая односвязная поверхность (возникающая там обычно как универсальная накрьвающая римановой поверхности) конформно отображается на $C \sim \mathbb{R}^{2}$ или на $H^{2} \sim B^{2}$, эти два случая различают как параболический и гиперболический конформный тип поверхности соответственно.

В высших размерностях конформных отображений на канонические области принципиально нет (в отличие от двумерного случая и в отличие от просто конформных замен метрики $g$ на $\lambda^{2} g$ ). Рассмотренные выше конформные инварианты позволяют, однако, дать внутреннее, конформно-инвариантное определение конформного типа риманова многообразия, не прибегая к конформным отображениям на канонические области.

Подобно пространствам Евклида и Лобачевского, произвольные некомпактные римановы (и даже субримановы) многообразия теперь можно конформно-инвариантно разделить на два класса в соответствии с тем, какова конформная емкость абсолюта (бесконечности) этого многоообразия (см. [73], [74]).

Некомпактное риманово многообразие $\left(M^{n}, g\right)$ будем назьвать многообразием конформно-параболического типа, если конформная емкость его абсолюта равна нулю (условно сар $R\left(C, \partial M_{\infty}^{n}\right)=0$ для любого отличного от точки континуума $C \subset M^{n}$ ); если же эта емкость положительна, то будем назьвать $\left(M^{n}, g\right)$ многообразием конформно-гиперболического типа.

Такое разделение многообразий по указанньм конформным типам, очевидно, инвариантно относительно конформных замен римановой метрики многообразия.

Конформный тип многообразия определяется лишь геометрией многообразия "на бесконечности" или, как говорят, асимптотической геометрией многообразия.

Конформная параболичность или гиперболичность многообразия (в соответствии с изложенным вьше) на другом язьке означает, что модуль семейства всех кривых на многообразии, выходяших на бесконечность (т.е. покидающих любую его компактную часть), равен нулю или положителен соответственно.

Заканчивая обсуждение понятия конформного типа риманова многообразия, отметим, что термины параболический и гиперболический ходят по всей математике и в разных ее отделах часто имеют разное содержание. Не следует, например, думать, что римановы многообразия конформно-гиперболического типа имеют отрицательную кривизну. Кривизна вообше не является конформньм инвариантом. Например, 
метрика пространства Лобачевского постоянной отрицательной кривизны в модели Пуанкаре конформна евклидовой плоской метрике. Последняя, в свою очередь, после стереографической проекции становится метрикой постоянной положительной кривизны.

Наконец, отметим, что в теории римановых поверхностей тип поверхности определяется как конформный тип ее универсальной накрьвающей. Например, крендель с проколом - это некомпактная поверхность, универсальная накрьвающая которой имеет конформно-гиперболический тип, хотя сама поверхность в нашей терминологии имеет конформно-параболический тип. В высших размерностях этого разногласия нет, а в двумерном случае его избегают, говоря, что риманова поверхность имеет нулевую или ненулевую границу (не путать с пустым и непустым множеством). В нашем примере крендель с проколом имеет нулевую границу (точнее, идеальную границу нулевой конформной емкости).

\section{7. Асимптотическая геометрия и конформный тип многообразия}

Вьше было дано формальное определение конформного типа риманова многообразия (или, скорее, вообще типа конформной структуры на нем). Вопрос о том, как по геометрии многообразия узнавать его конформный тип, принято (в соответствии с традицией, идущей от двумерного случая и теории римановых поверхностей) называть проблемой типа. Много работ разных периодов времени посвящено этой теме (см., например, [3], [75]-[79]), но до конща она так и не исчерпана (например, Милнор отмечает, что мы даже не всегда умеем по функции определить конформньй тип двумерной поверхности, являющейся графиком этой функции над всей плоскостью).

Мы остановимся здесь на двух критериях конформного типа риманова многообразия, которые можно рассматривать как развитие идей Альфорса, относящихся к проблеме типа римановых поверхностей. Заодно будет сформулирован и решен еше один чисто геометрический вопрос, на сей раз касающийся изопериметрического неравенства на римановом многообразии.

(Интересно, что близкие идеи и в тот же отрезок времени, что и у Альфорса, проявляются также в работе Лаврентьева [3], где проблема типа затрагивается в качестве одного примера приложений основного результата работы [3] - обобщенной теоремы Римана.)

Изопериметрическое неравенство - это соотношение вида

$$
P(V(D)) \leqslant S(\partial D)
$$

между объемом $V(D)$ области $D$ и площадью $S(\partial D)$ ее границы, где $P$ - функция, называемая изопериметрической функиией пространства. Конечно, интерес представляет только нетривиальная, а чаше всего даже максимальная изопериметрическая функция. Например, для евклидова $n$-мерного пространства $\mathbb{R}^{n}$ таковой, с точностью до множителя, является функшия $P(x)=x^{\frac{n-1}{n}}$, а для пространства Лобачевского $H^{n}$ - линейная функция $P(x)=x$.

Далее в качестве пространства выступает произвольное $n$-мерное некомпактное и без края риманово многообразие $M$ с некоторой исходной метрикой $g$. При переходе от метрики $g$ к конформной ей метрике $\widetilde{g}=\lambda^{2} g$ (где $\lambda$ - некоторая положительная регулярная функция на $M$ ) одноименные геометрические объекты будут наделяться тильдой. 
Будем считать метрику $g$ полной, а объем в ней всего многообразия бесконечным; этого, очевидно, всегда можно добиться, переходя, если надо, к конформно эквивалентной метрике.

Введем следующие обозначения: $B(r)$ - геодезический в метрике $g$ шар радиуса $r$ с фиксированным центром $x_{0} \in M ; V(r)$ - его объем $(n$-мера); $S(r)$ - плошадь $\left((n-1)\right.$-мера) его граничной сферы $\partial B(r) ; R_{r_{0}}^{r}$ - кольцевая область $B(r) \backslash \bar{B}\left(r_{0}\right)$, которую мы будем называть геодезическим кольцом или просто кольцом.

Двусторонняя оценка конформной емкости конденсатора и признаки конформного типа многообразия. Связь того, что уже сказано о проблеме типа, с тем, что мы собираемся изложить, в значительной степени осуществляют и проясняют следуюшие соотношения (см. [80]-[82] или [73], [74]):

$$
\left(\int_{V\left(r_{0}\right)}^{V\left(r_{1}\right)} P^{\frac{n}{1-n}}\right)^{1-n} \leqslant \operatorname{cap} R_{r_{0}}^{r_{1}} \leqslant\left(\int_{r_{0}}^{r_{1}} S^{\frac{1}{1-n}}\right)^{1-n} .
$$

Эти неравенства дают следующие геометрические достаточные (необходимые) признаки конформного типа риманова многообразия.

Для конформной гиперболичности (параболичности) п-мерного риманова многообразия $(M, g)$ достаточно (необходимо), чтобь сходился (расходился) интеграл $\int^{\bullet} P^{\frac{n}{1-n}}$.

Для конформной параболичности (гиперболичности) п-мерного риманова многообразия $(M, g)$ достаточно (необходимо), чтобь расходился (сходился) интеграл $\int^{\bullet} S^{\frac{1}{1-n}}$.

В обоих случаях здесь имеется в виду сходимость интеграла на верхнем пределе.

Полезно заметить, что если метрика $g$ сферически симметрична относительно центра $x_{0}$, т.е. если $g$ зависит только от расстояния $r$ до $x_{0}\left(\right.$ как в $\mathbb{R}^{n}$ и $\left.H^{n}\right)$, то последний из двух указанных признаков конформного типа становится критерием.

О критериях мы еше скажем ниже, а здесь отметим, что, обобщая результаты Кобаяси и Неванлинны, Альфорс в [75] (см. также [5]) показал, что расходимость интеграла $\int^{\bullet} S^{-1}$ является достаточным признаком параболичности односвязной открытой поверхности в классическом смысле теории униформизации, т.е. в смысле возможности ее конформного отображения на всю комплексную плоскость.

Альфорс отметил также, что в конформном классе метрики поверхности параболического типа всегда найдется такая метрика, в которой этот интеграл расходится (такую метрику в случае конформного отображения поверхности на плоскость можно снять прямо с плоскости).

Это красивое наблюдение может служить некоторым основанием для трансформации получаемого таким образом критерия параболичности в определение конформной параболичности любого $n$-мерного риманова многообразия, как это сделано в [83], где затем доказана эквивалентность такого определения приведенному вьше определению в терминах конформной емкости.

Итак, справедлив следуюший критерий Альфорса конформной параболичности риманова многообразия. 
Риманово многообразие $(M, g)$ размерности $n$ конформно-параболично тогда и только тогда, когда существует такая конформная $g$ метрика $\widetilde{g}$, в которой многообразие $(M, \widetilde{g})$ полно и $\int^{\infty} \widetilde{S}^{\frac{1}{1-n}}=\infty$.

Отметим, что Альфорс [5], по-видимому, был первым, кто привлек к проблеме типа и теории распределения значений изопериметрическое неравенство (см., однако, и приведенное выше замечание о работе Лаврентьева [3]) и для $n=2$ установил указанную взаимосвязь между крайними членами соотношения $(\star \star)$. Более того, он заметил, что слева вместо функции $P$ можно поставить любую функцию $\widetilde{P}$, изопериметрическую относительно метрики, конформной исходной метрике многообразия (пределами интегрирования должны теперь быть $\widetilde{g}$-объемы прежних областей). Это утверждение часто назьвают леммой Альфорса или, применительно к случаю произвольной размерности $n,-$ леммой Альфорса-Громова.

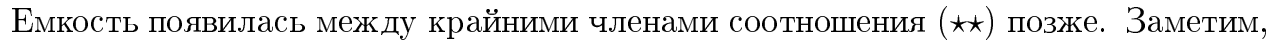
что как конформньй инвариант, в облике конформного модуля семейства кривых на плоскости, она родилась у Бёрлинга и Альфорса [68] и оформилась в метод экстремальных длин, которьй, в свою очередь, выкристаллизовался из приемов Грётша. (См. по этому поводу исторический комментарий Альфорса к главе 4 в книге [69], а также в двухтомном собрании его сочинений. Метод экстремальных длин или модулей и сам этот конформньй инвариант успешно используются как в геометрической теории функщий, так и за ее пределами. Вспомним, например, его применение к исследованию граничного поведения конформных и квазиконформных отображений [84], [25], [56] или к качественньм вопросам магнитной гидродинамики и теории зацеплений $[85],[86]$.

Приведем теперь развернутую формулировку сравнительно недавней теоремы [73], содержащей критерии конформной параболичности римановых многообразий и устанавливаюшей их взаимосвязь.

Условимся говорить, что некоторое свойство или соотношение реализуемо в каком-то классе метрик, если оно реализуется, т.е. вьполнено для некоторой метрики этого класса.

Например, любое риманово многообразие $(M, g)$ в конформном классе его метрики реализуемо как полное риманово многообразие (т.е. класс полных конформных $g$ метрик не пуст).

Теперь мы можем сформулировать теорему.

Риманово многообразие $(M, g)$ размерности $n$ имеет конформно-параболический тип тогда и только тогда, когда в классе полных конформных $g$ метрик реализуемы следующие әквивалентные между собой условия:

(i) $\operatorname{vol}(M)<\infty$,

(ii) $\int^{\infty} S^{\frac{1}{1-n}}(r) d r=\infty$,

(iii) $\int^{\infty}\left(\frac{r}{V(r)}\right)^{\frac{1}{n-1}} d r=\infty$,

(iv) $\liminf _{r \rightarrow \infty} \frac{V(r)}{r^{n}}<\infty$. 
Здесь, как и прежде, $V(r)$ и $S(r)$ - объем и площадь соответственно геодезического шара радиуса $r$ и его границы, a $\operatorname{vol}(M)$ - объем всего многообразия по отношению к метрике, существование которой утверждается в теореме.

Отметим, что в каждой индивидуальной метрике справедливы импликации

$$
\text { (i) } \Rightarrow \text { (iv) } \Rightarrow \text { (iii) } \Rightarrow \text { (ii). }
$$

Обратных импликаций в индивидуальной метрике может не быть. Теорема, однако, утверждает, что в рамках всего класса конформных метрик многообразия все эти соотношения (свойства) эквивалентны.

Приведение к линейному виду изопериметрической функции на многообразии конформно-гиперболического типа. Все, что присутствует в последней теореме, связано только с одним правым неравенством соотношения $(\star \star)$. В отличие от площадей и объемов, однозначно определяемых метрикой, изопериметрическая функция $P$ многообразия в левой части $(\star \star)$ имеет произвол. Разумеется, интерес представляет возможно большая (максимальная) изопериметрическая функция. Чтобы дать критерий конформного типа многообразия в терминах изопериметрической функции, желательно решить представляюший самостоятельньй интерес вопрос о том, к какому стандартному виду можно привести изопериметрическую функцию многообразия в пределах конформного класса его метрики.

Оказалось [87], что на любом римановом многообразии конформно-гиперболического типа конформной заменой метрики изопериметрическую функцию можно привести к тому же линейному виду, какой она имеет в каноническом гиперболическом пространстве Лобачевского.

Учитывая левое неравенство соотношения ( $\star)$, получаем следуюший критерий.

Некомпактное риманово многообразие имеет конформно-гиперболический тип тогда и только тогда, когда конформной заменой метрики на нем моэкно реализовать такое же линейное изопериметрическое неравенство $V(D) \leqslant$ $S(\partial D)$, как и в каноническом гиперболическом пространстве Лобачевского.

Здесь, как всегда, $V(D)$ - объем области $D$ на многообразии, а $S(\partial D)$ - площадь ее границы.

Есть рабочая гипотеза [87], что на $n$-мерном многообразии конформно-параболического типа всегда найдется такая полная метрика, конформно эквивалентная исходной метрике многообразия, по отношению к которой изопериметрическое неравенство имеет тот же вид, какой оно имеет в $n$-мерном пространстве Евклида.

Отметим, что все перечисленные результаты о конформном типе после соответствующей модификации переносятся и на субримановы многообразия (с неголономной структурой) [74], [88].

Мы не останавливаемся здесь более на асимптотической геометрии и геометрических признаках типа (в частности, на различных понятиях параболичности и гиперболичности) многообразия. Скажем лишш, что они представляют серьезный интерес в вопросах глобального анализа (римановы поверхности, свойства решений уравнений на многообразиях, спектры, динамика, диффузия, броуновское движение, .. . ). Помимо уже указанных работ, относившихся к проблеме конформного типа, см., например, обзор [81] и обширную библиограффию в нем. 


\section{8. Конформно-инвариантная форма теоремы о глобальном гомеоморфизме}

Теперь от конформной геометрии можно вновь вернуться к теореме о глобальном гомеоморфизме и придать ей форму, включаюшую как исходную теорему, так и ее обобщение, предложенное Громовым.

Если $f: M^{n} \rightarrow N^{n}$-квазиконформное погружение (иммерсия или локально гомеоморфное отображсене) многообразия $M^{n}$ конформно-параболического типа в односвязное риманово многообразие $N^{n}$ той жсе размерности $n$, то при $n \geqslant 3$ отображение является вложением (инбективно) и хаусдорфова размерность множсества $N^{n} \backslash f\left(M^{n}\right)$ равна нулю.

Чтобы полностью включить сюда и исходную теорему, утверждаюшую в случае $M^{n}=N^{n}=\mathbb{R}^{n}$, что $f\left(M^{n}\right)=N^{n}$ (тогда как в общем случае это не так), сделаем следующее замечание.

Пользуясь тем, что конформная емкость (модуль) является конформным инвариантом, который, к тому же, есть квазиинвариант квазиконформных отображений, можно ввести на многообразии (или вне некоторой его компактной части) такую конформно-инвариантную метрику, по отношению к которой квазиконформные отображения $f: M^{n} \rightarrow N^{n}$ многообразий становятся квазиизометриями. Если теперь пополнить многообразия по такой метрике, то отображение $f: M^{n} \rightarrow N^{n}$ естественно продолжится до отображения $\bar{f}: \bar{M}^{n} \rightarrow \bar{N}^{n}$ пополнененных пространств (см. обзор [61]).

Значит, предыдущую формулировку теоремы можно (или следует) дополнить, сказав, что полученное вложсене $f: M^{n} \rightarrow N^{n}$ продолжается до гомеоморфизма $\bar{f}: \bar{M}^{n} \rightarrow \bar{N}^{n}$.

В случае, когда $M^{n}=N^{n}=\mathbb{R}^{n}$, как это было в исходной теореме о глобальном гомеоморфизме, пространство $\mathbb{R}^{n}$ пополняется одной точкой $\left(\bar{R}^{n}=S^{n}\right)$, неподвижной при отображении $\bar{f}: S^{n} \rightarrow S^{n}$. Только это обстоятельство и позволяет утверждать, что $f\left(\mathbb{R}^{n}\right)=\mathbb{R}^{n}$. В обшем случае множество $N^{n} \backslash f\left(M^{n}\right)$ вьпускаемых значений непусто (взять хотя бы тождественное отображение проколотой сферы на эту же сферу без прокола).

Изолированная особенность. Теперь уместно обратить внимание на еще одну сторону дела. Из обших топологических соображений, связанных с индексом, вытекает, что если локально гомеоморфное отображение $f: \mathbb{R}^{n} \rightarrow \mathbb{R}^{n}$ (или $f: \dot{\mathbb{S}}^{n} \rightarrow \dot{\mathbb{S}}^{n}$ ) гомеоморфно в окрестности бесконечности, то оно гомеоморфно в целом. Поэтому представляет интерес поведение квазиконформного погружения в окрестности изолированной особой точки. На этот счет имеется следующий свежий результат [89], которьй, ввиду сказанного вьше, тоже можно рассматривать как обобшение теоремы о глобальном гомеоморфизме для квазиконформных погружений.

Пусть $M^{n}$ и $N^{n}$ - римановь многообразия размерности $n$, о - точка на $M^{n}$ и $\dot{M}^{n}:=M^{n} \backslash\left\{\right.$ o\}. Пусть $f: \dot{M}^{n} \rightarrow N^{n}-$ квазиконформное погружсние. Если многообразие $N^{n}$ односвязно, а $n \geqslant 3$, то отображение $f$ инвективно в некоторой проколотой окрестности точки о; при этом особенность либо устраняется непосредственно, либо этого можсно добиться, пополняя $N^{n}$ единственной точкой.

Говоря о погружении или иммерсии, мы, как и вьше, имеем в виду локально инъективное (локально обратимое) отображение. Квазиконформность погружения подра- 
зумевает ограниченность его коэффищиента квазиконформности. Устранение особенности предполагается в категории квазиконформных погружений.

Ранее это утверждение было известно только в случае $N^{n}=\mathbb{R}^{n}$ (см. [90], [91]). В основе доказательства сформулированной теоремы лежит теорема о продолжении ростка квазиконформного погружения из работы [92].

Теорема Пикара. В самом начале статьи, говоря о возникновении теории квазиконформных отображений, мы упомянули о том, что многие даже сравнительно тонкие факты теории голоморфных функций справедливы и для более широкого класса функций и отображений. В частности, это относится и к теореме Пикара. Мы добавим теперь к сказанному несколько обших соображений о природе теорем этого типа и закончим одним конкретным утверждением.

Пространство Лобачевского конформно вкладьвается в евклидово пространство, а евклидово пространство в пространство Лобачевского не погружается конформно. Это становится очевидньм, если вспомнить соответственно модель Пуанкаре гиперболического пространства и две теоремы Лиувилля (о том, что на плоскости целая ограниченная функция - постоянна, и о конформной жесткости областей пространства $\mathbb{R}^{n}$ при $\left.n>2\right)$.

Конформный тип многообразия инвариантен не только при конформных заменах метрики, но и при многих других действиях, в том числе при квазиконформных отображениях. Поскольку конформньй тип многообразия определяется только его асимптотической геометрией (поведением на бесконечности), то и от отображений, его сохраняюших, можно требовать, чтобы что-то вьполнялось асимптотически (на бесконечности), не заботясь о поведении на компактной части.

Суть многих утверждений типа теоремы Лиувилля (о постоянной) или теоремы Пикара, а также их обобшений, состоит в том, что в рассматриваемом классе преобразований (например, квазиконформных) нельзя параболический объект (с малой идеальной гранищей) погрузить в объект гиперболического типа (с массивным абсолютом). Малая граница скорее стирается, а не раздувается. Разумеется, на одни и те же веши по мере появления обобшаюших знаний появляется возможность смотреть с новых и даже разных позиций.

Возвращаясь к теореме Пикара, отметим, что на плоскости она верна не только для голоморфных функций и неоднолистных квазиконформных отображений (называемых иногда квазиголоморфными), но, например, и для отображений, коэффициент квазиконформности которых $k(r)$ в круге радиуса $r$ растет не слишком быстро с ростом $r$, а именно если $\int^{\infty} \frac{d r}{r k(r)}=\infty$ (см. [4]). Это условие нам уже встречалось вьше по близкому поводу и на самом деле оно и отвечает за сохранение параболического типа отображаемого многообразия.

Теорема Пикара-Риккмана (о которой мы уже говорили) относится к размерностям $n>2$ и отличается двумя особенностями: во-первых, выпускаемых точек может быть больше двух, а во-вторых (и это особенно контрастирует с плоским случаем, описанным вьше), количество выпускаемых точек может неограниченно расти с ростом коэффициента квазиконформности отображения.

Разумеется, все такие отображения обязаны иметь ветвления, что следует из теоремы о глобальном гомеоморфизме.

Если же рассматривать локально-гомеоморфные отображения, то можно доказать следуюшее контрастирующее с теоремой Пикара-Риккмана утверждение [92]. 
Квазиконформная иммерсия одного риманова многообразия в другое риманово многообразие той же размерности $n>2$ около существенно особой точки не имеет выпускаемых значений.

При некоторых дополнительных топологических ограничениях подобное явление наблюдается и в двумерном случае [93], [94]. Я признателен И. Кра, обратившему мое внимание на указанные работы.

\section{9. Некоторые открытые вопросы}

Нелинейные операторы. Пусть $f: D \rightarrow H$ - вообще говоря, нелинейньй гладкий оператор, действуюший из области $D$ гильбертова (или даже банахова) пространства $H$. Пусть касательный оператор $f^{\prime}(x)$ имеет непрерьвный обратный в каждой точке $x \in D$. Это обеспечивает локальную обратимость оператора $f$.

Вспоминая определение коэффищиента квазиконформности, в точке $x$ имеем

$$
k_{f^{\prime}(x)}:=\left\|f^{\prime}(x)\right\| \cdot\left\|\left(f^{\prime}(x)\right)^{-1}\right\|
$$

и, значит, понятия квазиконформности и коэффициента квазиконформности действуют и для операторов.

Тогда естественно спросить, верны ли в бесконечномерном случае для нелинейных операторов с ограниченньм искажением (квазиконформных операторов) следующие теоремы:

о глобальном гомеоморфизме,

о радиусе инбективности,

об изолированной особенности.

Например, операторньй аналог теоремы о глобальном гомеоморфизме давал бы следуюший принцип обратимости, или теорему сушествования и единственности, для нелинейных операторов.

Если оператор $f: H \rightarrow H$, действующий в гильбертовом пространстве $H$, локально обратим и имеет ограниченное искажение, то он глобально обратим.

Значит, в этом случае уравнение $f(x)=y$ разрешимо и единственным образом при любой правой части $y \in H$.

Очень может быть (как это случается в теории операторов), что свойства пространства (гильбертово, банахово) здесь существенны.

Напомним, что Неванлинна [95] доказал теорему Лиувилля о конформных отображениях и в случае гильбертова пространства. Однако я не знаю больше ни одного результата о собственно квазиконформных (не квазиизометрических) отображениях в бесконечномерном случае.

Асимптотика радиуса инъективности. В связи с поставленньми вьше вопросами естественно возникает вопрос, относящийся к классическому конечномерному случаю.

Какова асимптотика функиии $r(n, k)$ (радиуса инбективности) по размерности $n$, или, конкретнее, верно ли, что $\rho(k):=\inf _{n} r(n, k)>0$ и существует универсальный радиус ингективности $\rho(k)$ ? 
Стирание особенностей. Вьше (см. раздел "Изолированная особенность") было сказано, что если имеется квазиконформное погружение $f$ проколотой окрестности $U \backslash\{o\}$ точки $o \in M^{n}$ многообразия $M^{n}$ размерности $n>2$ в односвязное многообразие $N^{n}$ той же размерности, то $f$ является вложением (инъективно) в некоторой меньшей проколотой окрестности этой точки.

Есть основания полагать, что этот результат можно с пользой для дела заметно усилить. Однако до сих пор не решена поставленная еще в работе [57] следующая модельная задача.

Пусть $M^{n}=N^{n}=\mathbb{R}^{3}$, а вместо $U \backslash\{o\}$ рассматриваем проколотую окрестность $U \backslash\{I\}$ замкнутого отрезка $I$.

Верно ли, что квазиконформное погружение $f: U \backslash\{I\} \rightarrow \mathbb{R}^{3}$ проколотой окрестности отрезка является вложсением (инбективно) в некоторой меньшей проколотой окрестности отрезка $I$ ?

И второй (совсем свежий) вопрос касается связи неустранимости изолированной особенности и возможной при этом только очень специальной топологии многообразия образа.

Верно ли, что в размерностях $n>2$ изолированная существенно особая точка квазиконформного погружения риманова многообразия в другое риманово многообразие той же размерности может возникнуть, только если последнее имеет топологический тип $\mathbb{S}^{n-1} \times \mathbb{S}^{1}\left(\right.$ хопфов тор) или $\mathbb{T}^{k} \times \mathbb{R}^{n-k}$, где $\mathbb{T}^{k}-$ стандартный $k$-мерный тор?

Когда мы здесь говорим о существенной особенности, имеется в виду, что отображение не имеет в ней ни конечного, ни бесконечного предела. Бесконечность предела означает, что образ точки многообразия, по мере приближения точки многообразия к особенности, покидает любой компакт на многообразии, куда идет отображение.

Вопрос Новикова. В связи с вопросом Новикова о том, для каких многообразий любое квазиконформное погружение $f: M^{n} \rightarrow N^{n}$ автоматически оказьвается вложением, сделаем следующее замечание.

Мы видели, что если $n>2$ и $N^{n}$ односвязно, то конформной параболичности многообразия $M^{n}$ достаточно, чтобы это было так. Легко понять, что при этом многообразие $N^{n}$ тоже оказьвается многообразием конформно-параболического типа и конформно-инвариантные пополнения этих многообразий должны быть квазиконформно неразличимы.

Однако это достаточное условие еще не является необходимым. Группа Гейзенберга $H_{m}$ размерности $n=2 m+1$ (к примеру, трехмерная) с инвариантной римановой метрикой на ней имеет конформно-гиперболический тип (см. [96], [74]). Вместе с тем не сушествует даже неоднолистных квазиконформных отображений ее в себя, вьпускающих хотя бы одно значение [97]. То есть в этом случае справедливы и теорема Лиувилля, и теорема Пикара.

По-видимому, в этом случае справедлива и теорема о глобальном гомеомор$\oint и з м е$, хотя, например, для пространства Лобачевского она, конечно, не имеет места.

Более того, хотя оба пространства имеют конформно-гиперболический тип, нет никакого непостоянного однолистного или неоднолистного квазиконформного отображения групшы Гейзенберга в пространство Лобачевского той же топологической размерности [97], хотя в обратном направлении такое отображение, очевидно, есть. 
С этой ситуацией мы уже сталкивались, когда вместо групшы Гейзенберга выступало евклидово пространство. Оно имело конформно-параболический тип.

Таким образом, препятствием к отображению служит не только различие конформного типа.

Дело в том, что многообразия одного конформного типа в размерностях $n>2$ могут, тем не менее, иметь разную (квази)конформную структуру идеальной гранищы (абсолюта).

Например, если из сферы $\mathbb{S}^{3}$ удалить отрезок (в топологическом смысле) или площадку, то в обоих случаях получатся многообразия конформно-гиперболического типа. Однако они квазиконформно различны и первое нельзя квазиконформно поместить во второе, грубо говоря, потому, что малым абсолютом (образом отрезка) нельзя отделиться от абсолюта второго многообразия, а совпадать эти абсолюты не могут. С другой стороны, второе многообразие легко квазиконформно вкладьвается в перBoe.

Чтобы пояснить ситуацию с обсуждаемым вопросом, напомним, например, казалось бы много более конкретную, но трансцендентную задачу классификации (описания) квазиконформно различных областей в $\mathbb{R}^{n}$, гомеоморфных шару. Или задачу описания областей, допускаюших квазиконформное отображение на шар. Множество таких областей имеет естественную структуру пространства Тейхмюллера, однако на сей раз это пространство даже не сепарабельно (см., например, [56]).

Квазиконформная изотопия. Хорошо известно (см., например, [11]) и часто используется то обстоятельство, что любое квазиконформное отображение плоскости можно квазиконформно изотопировать в тождественное отображение, непрерьвно уменьшая при этом коэффициент квазиконформности (снятие напряжений).

Верно ли это и в высших размерностях для квазиконформных отображений евклидова пространства в себя?

См. в этой связи работы [98], [99], аппарат и результаты которых тут могут быть весьма полезны, а также работы [100], [101], где имеются частичные результаты в нужном направлении.

Отметим, что условие непрерывного уменьшения коэффициента квазиконформности здесь существенно. Иначе можно было бы использовать стандартную гомотопию Александера, которая сохраняет коэффициент квазиконформности, делая его равньм единице лишш в последний момент, когда отображение тождественно.

Субримановы многообразия. Наряду с касательным расслоением часто естественным образом выделяются те или иные его подрасслоения. Например, связи в механике или в теории управления вводят ограничения на возможные направления смещения системы из данного состояния. Специальные подрасслоения и связанные с ними объекты повсюду встречаются в многомерном комплексном анализе (комплексные касательные), в симплектической геометрии (лагранжевы многообразия), в термодинамике (адиабатические и другие специальные процессы изменения термодинамического состояния).

Часто подрасслоения возникают как нулевые пространства системы дифференциалных форм. Вопросам интегрируемости таких подрасслоений, как известно, посвящены теоремы Фробениуса.

Пусть в каждой точке пространства $\mathbb{R}^{3}$ выбрана двумерная плоскость, и пусть путь в пространстве считается допустимьм, если вектор скорости в каждый момент лежит 
в соответствуюшей выделенной плоскости. Возникает вопрос: всегда ли можно соединить точки пространства допустимым путем?

(В случае термодинамики, которой интересовался Каратеодори, это вопрос о возможности, точнее, невозможности адиабатической связи двух произвольных термодинамических состояний системы. Каратеодори дал оригинальную формулировку второго начала термодинамики, в терминах адиабатической недостижимости некоторых состояний, связав его с теорией пфаффовых форм, и вообще дал в известном смысле современное математическое изложение классической термодинамики; см. paботу [102] и инищиированную Планком работу [103].)

Если распределение плоскостей неголономно (неинтегрируемо), то пространство $\mathbb{R}^{3}$ оказьвается в указанном смысле связным. Тогда в нем можно ввести новую метрику, измеряя расстояния длиной кратчайшей допустимой кривой, соединяющей рассматриваемые точки. Такую метрику в этом примере и в аналогичной общей ситуащии называют (по понятным теперь соображениям и, кажется, с легкой руки Громова [104]) метрикой Карно-Каратеодори (С-С метрикой).

Возьмем теперь в $\mathbb{R}^{3}$ плоскость $z=0$ с ее естественной евклидовой метрикой. Будем считать ее подпространством пространства $T_{0} \mathbb{R}^{3}$, касательного к $\mathbb{R}^{3}$ в начале координат. Посмотрим на точки пространства $\mathbb{R}^{3}$ как на элементы группы Гейзенберга $H_{1}$. Разнесем выбранную в начале координат (в единице групш) плоскость по всей групе. Получим неголономное распределение гиперплоскостей в $\mathbb{R}^{3}$, задающих касательное подрасслоение коразмерности 1. В выделенных подпространствах касательных пространств в каждой точке имеется риманова метрика. Такие объекты в геометрии называют субримановыми многообразиями. На субримановых многообразиях определена длина допустимой кривой, и если пространство связно в смысле допустимых переходов, то в нем определена метрика Карно-Каратеодори, как это действительно имеет место в рассматриваемом случае группы Гейзенберга. Построенная метрика Карно-Каратеодори, очевидно, инвариантна относительно соответствующего (левого, правого) действия групш Гейзенберга. Метрика Карно-Каратеодори позволяет рассматривать хаусдорфовы меры геометрических объектов разной размерности. Заметим, что метрическая размерность полученного пространства уже не 3 , a 4. С этим обстоятельством связаны многие особенности групшы Гейзенберга как C-C (Карно-Каратеодори)-пространства. В частности, она теперь имеет конформно-параболический тип (подробности см. в [74]). Все описанные конструкции, конечно, можно повторить для группы Гейзенберга высших размерностей.

Место группы Гейзенберга по отношению к субримановым (контактным) многообразиям сопоставимо с местом пространства $\mathbb{R}^{n}$ по отношению к гладким многообразиям: это их локальная линеаризация.

Определение квазиконформности отображения сохраняет силу и по отношению к C-C метрике на групе Гейзенберга.

Верна ли теорема о глобальном гомеоморфизме на группе Гейзенберга, рассматриваемой как пространство Карно-Каратеодори?

В работе [74] сказано, к какой специальной лемме об оценке конкретного интеграла (отвечающего за емкость) можно привести ответ на этот вопрос. В случае евклидова пространства лемма доказана (см. [92]). 


\section{0. Заключительные замечания}

Мы затронули здесь только фрагмент теории квазиконформных отображений (в основном пространственной теории и в основном в геометрическом аспекте), но постарались, хоть и бегло, проследить развитие идей от первоисточников до их современной реализации и указать ряд новых задач, естественно выросших из найденных решений старых.

Мыне касались теоретико-функциональных вопросов, связанных с дифференщиальными свойствами квазиконформных отображений, а также методов нелинейных уравнений эллиптического типа, с успехом работающих в этой теории. Они представлены в инициировавших их систематическое использование работах Решетняка и его книге [105]. См. также статью [106] и книгу [107].

Из обзоров, посвященных иным аспектам теории квазиконформных отображений на разных стадиях ее развития, укажем [108]-[111].

Ряд теоретико-групповых, аналитических и геометрических вопросов, тесно примыкаюших к обсуждавшимся здесь вопросам асимптотической геометрии многообразий, можно найти в [66], [104], [112], [113].

\section{СПИСОК ЛИТЕРАТУРЫ}

[1] H. Grötzsch. Über die Verzerrung bei schlichten nichtkonformen Abbildungen und über eine damit zusammenhängende Erweiterung des Picardschen Satzes // Ber. Verh. Sächs. Akad. Wiss. Leipzig. 1928. V. 80. P. 503-507.

[2] H. Grötzsch. Über möglichst konforme Abbildungen von schlichten Bereichen // Ber. Verh. Sächs. Akad. Wiss. Leipzig. 1932. V. 84. P. 114-120.

[3] M. Lavrentieff. Sur une méthode géométrique dans la représentation conforme // Atti Congr. intern. mat., Bologna, 1928: Comm. sez. V. 3. Bologna: Zanichelli, 1930. P. 241-242.

[4] M. A. Lavrentieff. Sur une classe de représentations continues // Матем. сб. 1935. Т. 42. № 4. C. 407-424.

[5] L. Ahlfors. Zur Theorie der Überlagerungsflächen // Acta Math. 1935. V. 65. P. 157-194.

[6] O. Teichmüller. Untersuchungen über konforme und quasi-konforme Abbildungen // Deutsche Math. 1938. V. 3. P. 621-678.

[7] O. Teichmüller. Extremale quasikonforme Abbildungen und quadratische Differentiale // Abh. Preuß. Akad. Wiss. Math.-Natur. Kl. 1940. V. 22. P. 1-197.

[8] O. Teichmüller. Ein Verschiebungssatz der quasi-konformen Abbildungen // Deutsche Math. 1944. V. 7. P. 336-343.

[9] O. Teichmüller. Veränderliche Riemannsche Flächen // Deutsche Math. 1944. V. 7. P. 344-359.

[10] Л. Альфорс, Л. Берс. Пространства римановых поверхностей и квазиконформные отображения. М.: ИЛ, 1961. (Библ. сб. "Математика".)

[11] Л. Альфорс. Лекции по квазиконформным отображениям. М.: Мир, 1969.

[12] C.B. Morrey. On the solutions of quasi-linear elliptic partial differential equations // Trans. Amer. Math. Soc. 1938. V. 43. P. 126-166.

[13] М.А. Лаврентьев. Основная теорема теории квазиконформных отображений плоских областей // Изв. АН СССР. 1948. Т. 12. С. 513-554. (См. также М.А. Лаврентьев. Избранные труды. Математика и Механика. М.: Наука, 1990. С. 219-319.)

[14] И.Н. Векуа. Обобщенные аналитические функции. М.: Физматгиз, 1959.

[15] L. Ahlfors, L. Bers. Riemann's mapping theorem for variable metrics // Ann. of Math. (2). 1960. V. 72. № 2. P. 385-404.

[16] Р. Курант. Уравнения с частными производньми. М.: Мир, 1964.

[17] Дж. Милнор. Голоморфная динамика. Ижевск: НИЦ "Регулярная и хаотическая динамика", 2000.

[18] М. А. Лаврентьев. Об одном дифференциальном признаке гомеоморфных отображений трехмерных областей // Докл. АН СССР. 1938. Т. 20. С. 241-242. 
[19] А. И. Маркушевич. О некоторых классах непрерьвных отображений // Докл. АН CCCP. 1940. T. 28. C. 301-304.

[20] M.A. Kreines. Sur une classe de fonctions de plusieurs variables // Матем. сб. 1941. T. 9 (51). № 3. C. 713-720.

[21] М. А. Лаврентьев. Устойчивость в теореме Лиувилля // Докл. АН СССР. 1954. Т. 95. C. $925-926$.

[22] М.А. Лаврентьев. К теории пространственных отображений // Сиб. матем. журн. 1962. T. 3. № 5. C. $710-714$.

[23] М.А. Лаврентьев. К теории отображений трехмерных областей // Сиб. матем. журн. 1964. T. 5. № 3. C. 596-602.

[24] Б. В. Шабат. Метод модулей в пространстве // Докл. АН СССР 1960. Т. 130. C. $1210-1213$.

[25] Б.В. Шабат. К теории квазиконформных отображений в пространстве // Докл. АН CCCP. 1960. T. 132. C. $1045-1048$.

[26] Ю.Г. Решетняк. О конформных отображениях в пространстве // Докл. АН СССР. 1960. T. 130 . C. $1196-1198$.

[27] Ю. Г. Решетняк. Об устойчивости в теореме Лиувилля о конформных отображениях пространства // Докл. АН СССР. 1963. Т. 152. № 2. С. 286-287.

[28] П. П. Белинский. О непрерывности пространственных квазиконформных отображений и о теореме Лиувилля // Докл. АН СССР. 1962. Т. 147. № 5. С. 1003-1004.

[29] J. Väisälä. On quasiconformal mappings in space // Ann. Acad. Sci. Fenn. Ser. A I Math. 1961. V. 298. P. 1-36.

[30] J. Väisälä. On quasiconformal mappings of a ball // Ann. Acad. Sci. Fenn. Ser. A I Math. 1961. V. 304. P. 1-17.

[31] F.W. Gehring. Symmetrization of rings in space // Trans. Amer. Math. Soc. 1961. V. 101. P. 499-519.

[32] F.W. Gehring. Rings and quasiconformal mappings in space // Trans. Amer. Math. Soc. 1962. V. 103. P. 353-393.

[33] B. Fuglede. Extremal lenght and functional completion // Acta Math. 1957. V. 98. P. $171-219$

[34] C. Loewner. On the conformal capacity in space // J. Math. Mech. 1959. V. 8. P. 411-414.

[35] E. D. Callender. Hölder continuity of $n$-dimensional quasi-conformal mappings // Pacific J. Math. 1960. V. 10. P. 499-515.

[36] A. Mori. On quasi-conformality and pseudo-analyticity // Trans. Amer. Math. Soc. 1957. V. 84. P. 56-77.

[37] A. Beurling, L. Ahlfors. The boundary correspondence under quasiconformal mappings // Acta Math. 1956. V. 96. P. 125-142.

[38] В.А. Зорич. Соответствие границ при $Q$-квазиконформных отображениях шара // Докл. АН СССР. 1962. Т. 145. С. 1209-1212.

[39] Г. Д. Мостов. Квазиконформные отображения и жесткость гиперболических пространственных форм // Математика: сб. пер. 1972. Т. 16. №5. С. 105-157. (Оригинал: G.D. Mostow. Quasi-conformal mappings in $n$-space and the rigidity of hyperbolic space forms // Inst. Hautes Études Sci. Publ. Math. 1968. V. 34. P. 53-104.)

[40] G. D. Mostow. Strong rigidity of locally symmetric spaces. Princeton, NJ / Tokyo: Princeton University Press / University of Tokyo Press, 1973. (Ann. of Math. Stud. № 78.)

[41] Б. Риман. Сочинения. М.-Л.: Гостехиздат, 1948.

[42] J. Liouville. Extension au cas de trois dimensions de la question du tracé géographique // 6-е приложение к книге: G. Monge. Application de l'analyse à la géométrie. 5-ième édition, revue, corrigée et annotée par J. Liouville. Paris: Bachelier, 1850. P. 609-616.

[43] П.П. Белинский. Устойчивость в теореме Лиувилля о пространственных квазиконформных отображениях // Некоторые проблемы математики и механики: Труды конференции, посвященной 70-летию академика М.А. Лаврентьева. Ленинград: Наука, 1970. C. $88-102$.

[44] П. П. Белинский. О порядке близости пространственного квазиконформного отображения к конформному // Сиб. матем. журн. 1973. Т. 14. С. 475-483. 
[45] Ю. Г. Решетняк. Теорема Лиувилля о конформных отображениях при минимальных предположениях регулярности // Сиб. матем. журн. 1967. Т. 8. С. 835-840.

[46] Ю. Г. Решетняк. Теоремы устойчивости в геометрии и анализе. Новосибирск: Наука, 1982.

[47] А. П. Копылов. Устойчивость в $C$-норме классов отображений. Новосибирск: Наука, 1990.

[48] С. Улам. Нерешенные математические задачи. М.: Наука, 1964.

[49] J. Gevirtz. Stability of isometries on Banach spaces // Proc. Amer. Math. Soc. 1983. V. 89. P. $633-636$.

[50] F. John. Rotation and strain // Comm. Pure Appl. Math. 1961. V. 14. P. 391-413. (Collected papers. V. 2 / ed. J. Moser. Boston: Birkhäuser, 1985. P. 643-665.)

[51] В.А. Зорич. Принцип статики и устойчивость изометрий // Комплексный анализ в современной математике. М.: ФАЗИС, 2001. С. 3-17.

[52] М.А. Лаврентьев. Избранные труды. Математика и Механика. М.: Наука, 1990.

[53] В.А. Зорич. Граничные свойства одного класса отображений в пространстве // Докл. AH CCCP. 1963. T. 153. C. 23-26.

[54] В.А. Зорич. Взаимосвязь теоремы Кёбе и теории Каратеодори // Матем. заметки. 1971. T. 10. C. $399-406$.

[55] В.А. Зорич. Класс Каратеодори и пространственньй аналог теоремы Кёбе // Теория отображений, ее обобщения и приложения: Сборник научных трудов. Киев: Наукова думка, 1982. С. 91-101.

[56] F.W. Gehring, J. Väisälä. The coefficients of quasiconformality of domains in space // Acta Math. 1965. V. 114. P. 1-70.

[57] В.А. Зорич. Теорема М. А. Лаврентьева о квазиконформных отображениях пространства // Матем. сб. 1967. Т. 74 (116). С. 417-433.

[58] S. Rickman. On the number of omitted values of entire quasiregular mappings // J. Anal. Math. 1980. V. 37. P. 100-117.

[59] S. Rickman. The analogue of Picard's theorem for quasiregular mappings in dimension three // Acta Math. 1985. V. 154. P. 195-242.

[60] S. Rickman. Quasiregular Mappings. Berlin: Springer-Verlag, 1993.

[61] V.A. Zorich. The global homeomorphism theorem for space quasiconformal mappings, its development and related open problems // Lecture Notes in Math. 1992. V. 1508. P. 132-148.

[62] В. А. Зорич. Допустимый порядок роста коэффициента квазиконформности в теореме М. А. Лаврентьева // Докл. АН СССР. 1968. Т. 181. С. 530-533.

[63] O. Martio, S. Rickman, J. Väisälä. Topological and metric properties of quasiregular mappings // Ann. Acad. Sci. Fenn. Ser. A I Math. 1971. V. 488. P. 1-31.

[64] F. John. On quasi-isometric mappings. II // Comm. Pure Appl. Math. 1969. V. 22. P. 41-66.

[65] M. Gromov. Hyperbolic manifolds, groups and actions // Riemann surfaces and related topics: Proceedings of the 1978 Stony Brook Conference. Princeton, NJ: Princeton Univ. Press, 1981. P. 183-213. (Ann. of Math. Stud. V. 97.)

[66] M. Gromov with appendices by M. Katz, P. Pansu, S. Semmes. Metric Structures for Riemannian and Non-Riemannian Spaces. Boston: Birkhäuser, 1999. (Progr. Math. V. 152.)

[67] V.A. Zorich. On Gromov's geometric version of the global homeomorphism theorem for quasiconformal mappings // XIV Rolf Nevanlinna Colloquium, Helsinki, June 10-14, 1990. Abstracts. P. 36.

[68] L. Ahlfors, A. Beurling. Conformal invariants and function-theoretic null-sets // Acta Math. 1950. V. 83. P. 101-129.

[69] L. Ahlfors. Conformal invariants. Topics in Geometric Function Theory. New York: McGraw-Hill, 1973. (См. также Lars Valerian Ahlfors, Collected Papers. V. 1, 2. Boston: Birkhäuser, 1982.)

[70] J. Väisälä. Lectures on $n$-Dimensional Quasiconformal Mappings. Berlin: Springer-Verlag, 1971. (Lecture Notes in Math. V. 229.)

[71] F.W. Gehring. Extremal lenght definitions for the conformal capacity of rings in space // Michigan Math. J. 1962. V. 9. P. 137-150. 
[72] W.P. Ziemer. Extremal lenght as a capacity // Michigan Math. J. 1970. V. 17. P. $117-128$.

[73] В.А. Зорич, В.М. Кесельман. О конформном типе риманова многообразия // Функц. анализ и его прил. 1996. Т. 30. № 2. С. 40-55.

[74] V.A. Zorich. Asymptotic geometry and conformal types of Carnot-Carathéodory spaces // Geom. Funct. Anal. 1999. V. 9. № 2. P. 393-411.

[75] L. Ahlfors. Sur le type d'une surface de Riemann // C. R. Acad. Sci. Paris Sér. A. 1935. V. 201. P. 30-32.

[76] L. Ahlfors, E. Calabi, M. Morse, L. Sario, D. Spencer (Eds). Contributions to the Theory of Riemann Surfaces. Centennial celebration of Riemann's dissertation. Princeton: Princeton Univ. Press, 1953. (Ann. of Math. Stud. № 30.)

[77] L. Ahlfors, L. Sario. Riemann Surfaces. Princeton: Princeton Univ. Press, 1960. (Princeton Math. Ser. V. 26.)

[78] J. Milnor. A note on curvature and fundamental group // J. Differential Geom. 1968. V. 2. P. 1-7.

[79] J. Milnor. On deciding whether a surface is parabolic or hyperbolic // Amer. Math. Monthly. 1977. V. 84. № 1. P. 43-46.

[80] В. Г. Мазья. Пространства С. Л. Соболева. Л.: Изд-во ЛГУ, 1985.

[81] A. Grigor'yan. Analytic and geometric background of recurrence and non-explosion of the Brownian motion on Riemannian manifolds // Bull. Amer. Math. Soc. (N.S.). 1999. V. 36. № 2. P. 135-249.

[82] A. Grigor'yan. Isoperimetric inequality and capacities on Riemannian manifolds // Oper. Theory Adv. Appl. 1999. V. 109. P. 139-153.

[83] R. Grimaldi, P. Pansu. Sur la croissance du volume dans une classe conforme // J. Math. Pures Appl. 1992. V. 9 (71). № 1. P. 1-19.

[84] E. Schlesinger. Conformal invariants and prime ends // Amer. J. Math. 1958. V. 80. P. 83-102.

[85] M.H. Freedman, Z.-X. He. Divergence free fields: Energy and asymptotic crossing number // Ann. of Math. (2). 1991. V. 134. № 1. P. 189-229.

[86] M.H. Freedman, Z.-X. He. Links of tori and the energy of incompressible flows // Topology. 1991. V. 30. № 2. P. 283-287.

[87] В.А. Зорич, В. М. Кесельман. Изопериметрическое неравенство на римановых многообразиях конформно-гиперболического типа // Функц. анализ и его прил. 2001. Т. 35. № 2. C. $12-23$.

[88] В.А. Зорич, В. М. Кесельман. Изопериметрическое неравенство на субримановых многообразиях конформно-гиперболического типа // УМН. 2000. Т. 55. № 6. С. 137-138.

[89] В.А. Зорич. Устранимая особенность квазиконформного погружения // УМН. 2001. T. 56. № 4. C. $147-148$.

[90] S. Agard, A. Marden. A removable singularity theorem for local homeomorphisms // Indiana Univ. Math. J. 1970. V. 20. P. 455-461.

[91] В.А. Зорич. Изолированная особенность отображений с ограниченным искажением // Матем. сб. 1970. Т. 81. С. 634-638.

[92] В. А. Зорич. Квазиконформные погружения римановых многообразий и теорема пикаровского типа // Функц. анализ и его прил. 2000. Т. 34. № 3. С. 37-48.

[93] M. Ohtsuka. On the behaviour of an analytic function about an isolated boundary point // Nagoya Math. J. 1952. V. 4. P. 103-108.

[94] H. Royden. The Picard theorem for Riemann surfaces // Proc. Amer. Math. Soc. 1984. V. 90. № 4. P. 571-574.

[95] R. Nevanlinna. On differentiable mappings // Princeton Math. Ser. 1960. V. 24. P. 3-9.

[96] P. Pansu. An isoperimetric inequality on the Heisenberg group // Proceedings of the conference "Differential Geometry on Homogeneous Spaces", Torino, 1983. P. 159-174.

[97] I. Holopainen, S. Rickman. Quasiregular mappings, Heisenberg group, and Picard's theorem // Proceedings of the Fourth Finnish-Polish Summer School in Complex Analysis, Jyväskylä, Finland, 1992 / ed. J. Lawrynowicz et al. Jyväskylä: Mathematisches Institut, Jyväskylä Univ., 1993. P. 25-35. (Ber. Univ. Jyväskylä Math. Inst. V. 55.)

[98] S. K. Donaldson, D. P. Sullivan. Quasiconformal 4-manifolds // Acta Math. 1989. V. 163. № 3/4. P. 181-252. 
[99] T. Iwaniec, G. Martin. Quasiregular mappings in even dimensions // Acta Math. 1993. V. 170. № 1. P. 29-81.

[100] И. В. Абрамов, Е. А. Роганов. Квазиконформные гомотопии простейших пространственных отображений // Матем. сб. 1989. Т. 180. № 10. С. 1347-1354.

[101] О.А.Асадчий. К принципу максимума для пространственных квазиконформных отображений // Матем. заметки. 1991. Т. 50. №6. С. 14-23.

[102] C. Carathéodory. Untersuchungen über die Grundlagen der Thermodynamik // Math. Ann. 1909. V. 67. P. 355-386. (См. также C. Carathéodory. Gesammelte mathematische Schriften. V. II. München: C. H. Beck'sche Verlagsbuchhandlung, 1955. P. 131-166. Пер. на русск. яз.: К. Каратеодори. Об основах термодинамики // Развитие современной физики. М.: Наука, 1964. С. 188-228.)

[103] C. Carathéodory. Über die Bestimmung der Energie und der absoluten Temperature mit Hilfe von reversiblen Prozessen // Sitzungsber. Preuß. Akad. Wiss. Phys.-Math. Kl. Berlin. 1925. P. 39-47. (См. также C. Carathéodory. Gesammelte mathematische Schriften. München: C. H. Beck'sche Verlagsbuchhandlung, 1955. P. 167-177.)

[104] M. Gromov. Carnot-Carathéodory spaces seen from within // Sub-Riemannian Geometry / ed. Bellaïche et al. Basel: Birkhäuser, 1996. P. 79-323. (Progr. Math. V. 144.)

[105] Ю. Г. Решетняк. Пространственные отображения с ограниченным искажением. Новосибирск: Наука, 1982.

[106] T. Iwaniec. $L^{p}$-Theory of quasiregular mappings // Lecture Notes in Math. 1992. V. 1508. P. 39-64.

[107] J. Heinonen, T. Kilpeläinen, O. Martio. Nonlinear Potential Theory of Degenerate Elliptic Equations. Oxford: Clarendon Press, 1993. (Oxford Math. Monographs.)

[108] J. Väisälä. A survey of quasiregular maps in $\mathbb{R}^{n}$ // Proc. Internat. Congr. Math., Helsinki, 1978. V. 2. Helsinki: Acad. Sci. Fennica, 1980. P. 685-691.

[109] S. Rickman. Recent advances in the theory of quasiregular maps // Proceedings of the 19th Nordic Congress of Mathematicians, Reykjavik, 1984, 1985. P. 116-125.

[110] P. Pansu. Quasiconformal mappings and manifolds of negative curvature // Proceedings of the 17th International Taniguchi Symposium "Curvature and Topology of Riemannian Manifolds", Katata, Japan, 1985. Berlin: Springer-Verlag, 1986. P. 212-229. (Lecture Notes in Math. V. 1201.)

[111] F.W. Gehring. Topics in quasiconformal mappings // Proc. Internat. Congr. Math., Berkeley, California, 1986. V. 1. Providence, RI: Amer. Math. Soc., 1987. P. 62-80.

[112] N. Varopoulos, L. Saloff-Coste, T. Coulhon. Analysis and Geometry on Groups. Cambridge: Cambridge Univ. Press, 1992.

[113] T. Coulhon, I. Holopainen, L. Saloff-Coste. Harnack inequality and hyperbolicity for subelliptic $p$-Laplacians with applications to Picard type theorem // Geom. Funct. Anal. 2001. V. 11. № 6. P. 1139-1191. 